\title{
Unfolding of the electronic structure through the induced representations of space groups: Application to Fe-based superconductors
}

\author{
Milan Tomićf Harald O. Jeschke, and Roser Valentí \\ Institut für Theoretische Physik, Goethe-Universität Frankfurt, \\ Max-von-Laue-Straße 1, 60438 Frankfurt am Main, Germany
}

(Dated: September 12, 2014)

\begin{abstract}
We revisit the problem that relevant parts of bandstructures for a given cell choice can reflect exact or approximate higher symmetries of subsystems in the cell and can therefore be significantly simplified by an unfolding procedure that recovers the higher symmetry. We show that bandstructure unfolding can be understood as projection onto induced irreducible representations of a group obtained by extending the original group of translations with a number of additional symmetry operations. The resulting framework allows us to define a generalized unfolding procedure which includes the point group operations and can be applied to any quantity in the reciprocal space. The unfolding of the Brillouin zone follows naturally from the properties of the induced irreducible representations. In this context, we also introduce a procedure to derive tight-binding models of reduced dimensionality by making use of point group symmetries. Further, we show that careful consideration of unfolding has important consequences on the interpretation of angle resolved photoemission experiments. Finally, we apply the unfolding procedure to various representative examples of Fe-based superconductor compounds and show that the one iron picture arises as an irreducible representation of the glide mirror group and we comment on the consequences for the interpretation of one-iron versus two-iron Brillouin zone representations.

PACS numbers: 71.15.Mb, 71.18.+y, 71.27.+a, 74.70.Xa
\end{abstract}

\section{INTRODUCTION}

Ab initio electronic structure calculations have become a primary tool of research for understanding the microscopic behavior of solids. The multitude of methods that have emerged to deal with periodic crystal systems, such as density functional theory (DFT) 1], rely on the Bloch theorem[2] in one form or the other. The Bloch theorem builds upon the translational symmetry of the crystal lattice and paves the way to the fundamental concepts needed to understand the electronic structure of periodic systems, such as the classification of the electronic states in terms of wavevectors $\boldsymbol{k}$ and the notion of bandstructure. Many important properties of the crystal, such as magnetic or transport properties, are encoded in the bandstructure. In addition, the bandstructure is important for the interpretation of a few experimental measurements like angle resolved photoemission spectroscopy (ARPES).

However, a problem arises in the practical use of DFT calculations whenever we have to deal with systems where the original translational symmetry is broken. These situations are encountered, for example, in calculations on doped materials or in magnetically ordered systems. Often, in these situations we have to employ large supercells whose size determines the periodicity of the bandstructure through the Bloch theorem. This results in a complicated bandstructure consisting of many bands, which is hard to interpret.

Recently, a number of methods have emerged [3 10 to alleviate this problem. A common approach shared among these methods, implicitly or explicitly, is a trans- formation from one Bloch basis to another.

An important aspect of the Bloch theorem is that it is an expression of one of the fundamental group-theoretical principles, which states that the eigenstates of a physical system can be classified according to the irreducible representations of its group of symmetries [11. In light of this, the bandstructure unfolding can be viewed as a transformation between the irreducible representations of different translation groups. Despite the number of recent publications [3], a rigorous consideration of the group-theoretical aspects of the bandstructure unfolding is still missing and as a consequence certain important properties are overseen, especially in relation to ARPES experiments.

The purpose of this work is to attempt to close this conceptual gap by introducing a bandstructure unfolding based on group theory. This treatment allows us to incorporate point group symmetries into a unified framework and generalizes the idea of using glide-mirror operations, initially proposed in the context of $\mathrm{LaFeAsO}$ [12], in order to obtain models of reduced dimensionality. Our bandstructure unfolding also allows for a clear understanding of the two-Fe versus one-Fe description of the electronic properties of Fe-based superconductors.

We will show that bandstructure unfolding can be achieved by projecting the bands onto the induced irreducible representations of the supergroup of the initial group of translations. We will also show that this leads naturally to the concept of the unfolded Brillouin zone. With the help of the point group operations, bandstructures can be unfolded beyond the limits of translational symmetry. Further, tight-binding models with reduced 
number of orbitals can be formulated under certain conditions.

The group-theoretical formulation of the unfolding procedure in terms of projections onto the irreducible subspaces allows us to unfold any quantity in the reciprocal space if we know how it behaves under the symmetry operations of the crystal lattice. In addition, the unfolding artifacts such as "ghost-bands" [6] or "incomplete bands" 13] are naturally explained as bands with projections onto the multiple irreducible subspaces in cases with broken symmetry.

\section{METHOD}

\section{Group of translations}

One of the fundamental statements in solid state physics is that the eigenstates of the Hamiltonian of a periodic system can be classified according to the irreducible representations of the group of translational symmetries of the system.

Let us denote the crystal lattice as $\mathcal{L}=\left\{\boldsymbol{R}_{\boldsymbol{n}}=\sum_{i=1}^{3} n_{i} \boldsymbol{a}_{i} \mid n_{i}=1, \ldots, N_{i}\right\}, \quad$ where $\boldsymbol{n}=\left(n_{1}, n_{2}, n_{3}\right), \quad N=N_{1} N_{2} N_{3}$ is the number of unit cells in the lattice, $\boldsymbol{R}_{\boldsymbol{n}}$ are their position vectors, and $\boldsymbol{a}_{i}$ are the vectors spanning the unit cell. The lattice is invariant under the action of the group of translation operators $\mathbb{T}=\left\{\hat{T}_{\boldsymbol{n}}\right\}$, such that $\boldsymbol{R}_{\boldsymbol{n}}+\boldsymbol{R}_{\boldsymbol{m}}=\hat{T}_{\boldsymbol{m}} \boldsymbol{R}_{\boldsymbol{n}}$, where periodic boundary conditions $\hat{T}_{N_{i}}=1$ are assumed. The translation group $\mathbb{T}$ is an Abelian cyclic group generated by the three generators $\hat{T}_{\boldsymbol{a}_{i}}$. As such, its irreducible representations are one-dimensional and given by $\Delta^{(\boldsymbol{k})}\left(\hat{T}_{\boldsymbol{n}}\right)=\exp \left(-\mathrm{i} \boldsymbol{k} \cdot \boldsymbol{R}_{\boldsymbol{n}}\right)$. There are $N$ inequivalent irreducible representations of $\mathbb{T}$ and they can be enumerated by the vector index $\boldsymbol{k} \in \mathcal{B}(\mathcal{L})$, where $\mathcal{B}(\mathcal{L})=\left\{\sum_{i=1}^{3} g_{i} \boldsymbol{b}_{i} \mid g_{i} \in[0,1)\right\}$ is the Brillouin zone (BZ), and $\boldsymbol{b}_{i}=2 \pi \varepsilon_{i j k} \boldsymbol{a}_{j} \times \boldsymbol{a}_{k} /\left[\boldsymbol{a}_{1} \cdot\left(\boldsymbol{a}_{2} \times \boldsymbol{a}_{3}\right)\right]$ are unit vectors of the reciprocal lattice $\mathcal{L}^{-1}$. To each irreducible representation $\Delta^{(\boldsymbol{k})}$ corresponds a one dimensional subspace $\mathbb{V}_{\boldsymbol{k}}$, defined as a co-domain of the projection operator

$$
\begin{aligned}
\hat{P}_{\boldsymbol{k}} & =\frac{1}{\sqrt{N}} \sum_{\boldsymbol{n}}\left[\Delta^{(\boldsymbol{k})}\left(\hat{T}_{\boldsymbol{n}}\right)\right]^{\dagger} \hat{T}_{\boldsymbol{n}} \\
& =\frac{1}{\sqrt{N}} \sum_{\boldsymbol{n}} \exp \left(\mathrm{i} \boldsymbol{k} \cdot \boldsymbol{R}_{\boldsymbol{n}}\right) \hat{T}_{\boldsymbol{n}}
\end{aligned}
$$

Because for all $\boldsymbol{n},\left[\hat{T}_{\boldsymbol{n}}, \hat{H}\right]=0$, the subspaces $\mathbb{V}_{\boldsymbol{k}}$ will be orthogonal irreducible subspaces of the Hamiltonian $\hat{H}$, and thus, a symmetry classification of the eigenstates of the Hamiltonian is achieved.

In order to proceed, we assume that we have $P$ localized electronic states, $|\mathbf{0}, \mu\rangle, \mu=1, \ldots, P$, centered at positions $\boldsymbol{s}_{\mu}$, occupying the unit cell located at origin $\mathbf{0}$. The sites $\boldsymbol{s}_{\mu}$ don't necessarily have to be different since we can consider cases with multiple orbitals per atomic site. The translationally invariant electronic states of the crystal lattice are obtained by the action of $\mathbb{T}$ onto these states, and the resulting localized electronic states are $\left|\boldsymbol{R}_{\boldsymbol{n}}, \mu\right\rangle=\hat{T}_{\boldsymbol{n}}|\mathbf{0}, \mu\rangle$ at positions $\boldsymbol{R}_{\boldsymbol{n}}+\boldsymbol{s}_{\mu}$. Application of the projector (1) to the states $|\mathbf{0}, \mu\rangle$ results in the familiar Bloch-states

$$
|\boldsymbol{k}, \mu\rangle=\frac{1}{\sqrt{N}} \sum_{\boldsymbol{n}} \exp \left(\mathrm{i} \boldsymbol{k} \cdot \boldsymbol{R}_{\boldsymbol{n}}\right)\left|\boldsymbol{R}_{\boldsymbol{n}}, \mu\right\rangle
$$

Using (2) as a basis, the Hamiltonian $\hat{H}$ is brought to the block-diagonal form, with $N$ blocks of size $P \times P$, whose elements are given by $\left[\hat{H}_{\boldsymbol{k}}\right]_{\mu \nu}=\langle\boldsymbol{k}, \mu|\hat{H}| \boldsymbol{k}, \nu\rangle$. Every block $\hat{H}_{\boldsymbol{k}}$ can be diagonalized separately, yielding a set of $P$ bands at $\boldsymbol{k}$. A set of bands for every $\boldsymbol{k} \in \mathcal{B}(\mathcal{L})$ represents the bandstructure for the given lattice $\mathcal{L}$.

\section{Extension to additional symmetry operations}

In many applications, the translation group $\mathbb{T}$ can be expanded with a certain number of operations, which are, approximately, symmetry operations of the lattice $\mathcal{L}$. When this is the case, we will have a new group $\mathbb{S}$, such that $\mathbb{T}$ is an invariant subgroup of $\mathbb{S}$, denoted $\mathbb{T} \triangleleft \mathbb{S}$. Owing to this fact, irreducible representations of $\mathbb{S}$ can be induced from $\mathbb{T}$ in a simple manner. Furthermore, addition of every new symmetry operation will halve the number of independent states $|\boldsymbol{k}, \mu\rangle$ at every $\boldsymbol{k}$, since additional operations will produce one half of the states $|\boldsymbol{k}, \mu\rangle$ from another half, and thus will halve the number of bands, producing the unfolded bandstructure. This is subject to certain conditions which will be outlined in the discussion that follows.

Let us assume now that we are expanding $\mathbb{T}$ with $K$ operations $\hat{C}_{i}$, denoted in Seitz notation [14, 15] as $\hat{C}_{i}=$ $\left(\hat{U}_{i} \mid \boldsymbol{\tau}_{i}\right), i=1, . ., K$, where $\hat{U}_{i}$ is a point group operation and $\tau_{i}$ is a fractional translation (with respect to the translations $\hat{T}_{\boldsymbol{n}}$ of $\mathbb{T}$ ) so that the combination of $\hat{C}_{i}$ and $\hat{T}_{\boldsymbol{n}}$ leads to a space group $\mathbb{S}$. Operations $\hat{C}_{i}$ are allowed to be pure translations but not pure point group operations, since in the case of the pure point group operations, we would not be able to interpret the unfolded bandstructure in terms of a lattice of reduced periodicity.

The action of $\hat{C}_{i}$ on an arbitrary point in the Cartesian space is given by

$$
\hat{C}_{i} \boldsymbol{r}=\hat{U}_{i} \boldsymbol{r}+\boldsymbol{\tau}_{i}
$$

while the combined action of $\hat{C}_{i}$ and $\hat{T}_{\boldsymbol{n}}$ results in the space group operation $\hat{S}_{\boldsymbol{n} i}$ defined as

$$
\hat{S}_{\boldsymbol{n} i} \boldsymbol{r}=\hat{T}_{\boldsymbol{n}} \hat{C}_{i} \boldsymbol{r}=\hat{U}_{i} \boldsymbol{r}+\boldsymbol{R}_{\boldsymbol{n}}+\boldsymbol{\tau}_{i}=\left(\hat{U}_{i} \mid \boldsymbol{R}_{\boldsymbol{n}}+\boldsymbol{\tau}_{i}\right) \boldsymbol{r}
$$

The space group operators $\hat{S}_{\boldsymbol{n} i}$ induce an action on the localized states. Under the point group operations $\hat{U}_{i}$, 
and fractional translations $\boldsymbol{\tau}_{i}$, states $\left|\boldsymbol{R}_{\boldsymbol{n}}, \mu\right\rangle$ transform into each other as $\left(\hat{U}_{i} \mid \boldsymbol{\tau}_{i}\right)\left|\boldsymbol{R}_{\boldsymbol{n}}, \mu\right\rangle=\sum_{\nu}\left|\boldsymbol{R}_{\boldsymbol{n}}, \nu\right\rangle W_{\nu \mu}\left(\hat{C}_{i}\right)$, where the matrices $\hat{W}\left(\hat{C}_{i}\right)$ represent the action of the operations $\hat{C}_{i}=\left(\hat{U}_{i} \mid \boldsymbol{\tau}_{i}\right)$ in the basis composed of the states $\left|\boldsymbol{R}_{\boldsymbol{n}}, \mu\right\rangle$. Their matrix elements can be written as $W_{\gamma \delta}\left(\hat{C}_{i}\right)=r_{\gamma \delta} \delta\left(\boldsymbol{s}_{\delta}-\hat{C}_{i} \boldsymbol{s}_{\gamma}\right)$, where $\delta$ is the Kronecker delta and sites $\boldsymbol{s}_{\delta}$ and $\hat{C}_{i} \boldsymbol{s}_{\gamma}$ are considered equal if they differ by a lattice vector. The total action of $\hat{S}_{n i}$ is

$$
\hat{S}_{\boldsymbol{n} i}\left|\boldsymbol{R}_{\boldsymbol{m}}, \mu\right\rangle=\sum_{\nu}\left|\boldsymbol{R}_{\boldsymbol{m}}+\boldsymbol{R}_{\boldsymbol{n}}, \nu\right\rangle W_{\nu \mu}\left(\hat{C}_{i}\right)
$$

The task is now to induce the irreducible representations of the space group $\mathbb{S}$. We first note that the operators $\hat{C}_{i}$ are the right coset representatives of $\mathbb{S}$ with respect to $\mathbb{T}$. Let the factor group, corresponding to this right coset decomposition be $\mathbb{C}=\mathbb{S}: \mathbb{T}$. The well known property of space groups is that every space group is solvable, that is, every space group can be decomposed into a series $\mathbb{S}_{0} \triangleleft \mathbb{S}_{1} \triangleleft \ldots \triangleleft \mathbb{S}_{D}=\mathbb{S}$, where every factor group in the decomposition is Abelian. In addition to that, it is always possible to find the decomposition series where the factor groups $\mathbb{C}_{i}=\mathbb{S}_{i+1}: \mathbb{S}_{i}$ are cyclic groups of index two or three. This simplifies the induction procedure further, since in case the factor group $\mathbb{C}$ is not a cyclic group, we can always decompose it into a subgroup series $\mathbb{C}_{0} \triangleleft \mathbb{C}_{1} \triangleleft \ldots \triangleleft \mathbb{C}_{B}=\mathbb{C}$, where every $\mathbb{C}_{i}$ is a cyclic group.

In the induction procedure, from every irreducible representation of $\mathbb{T}$, multiple irreducible representations of $\mathbb{S}$ can be induced. Some of these irreducible representations will be one-dimensional, while some will be multidimensional. The multi-dimensional induced irreducible representations of $\mathbb{S}$ will mix irreducible representations of $\mathbb{T}$ with different $\boldsymbol{k}$. Since our goal is to perform the unfolding within the same $\boldsymbol{k}$, we have to restrict ourselves only to the cases where the induction procedure yields one-dimensional irreducible representations of $\mathbb{S}$.

\section{One-dimensional irreducible representations of $\mathbb{S}$. Unfolding procedure}

For every irreducible representation $\Delta^{(\boldsymbol{k})}$ of $\mathbb{T}$, irreducible representations of $\mathbb{S}$ are determined from the little co-group $\mathbb{L}_{k}[14,15$. The little co-group is a group of all point group operations $\hat{U}_{i}^{k}$ such that $\hat{U}_{i}^{k} \boldsymbol{k}=\boldsymbol{k}+\boldsymbol{K}$, where $\boldsymbol{K}$ is a reciprocal lattice vector. Given an irreducible representation $\Lambda^{(\boldsymbol{k}, \alpha)}$ of $\mathbb{L}_{\boldsymbol{k}}$, an irreducible representation $\Delta^{(\boldsymbol{k}, \alpha)}$ of $\mathbb{S}$ is induced, where the coset representatives $\hat{C}_{i}$ are represented by

$$
\Delta^{(\boldsymbol{k}, \alpha)}\left(\left(\hat{U}_{i} \mid \boldsymbol{\tau}_{i}\right)\right)=\exp \left(-\mathrm{i} \boldsymbol{k} \cdot \boldsymbol{\tau}_{i}\right) \Lambda^{(\boldsymbol{k}, \alpha)}\left(\hat{U}_{i}\right)
$$

and the index $\alpha$ runs over all irreducible representations. This holds across the entire interior of the BZ, with the exception of the BZ boundary for the cases of the symmorphic space groups, where the induction procedure is a)
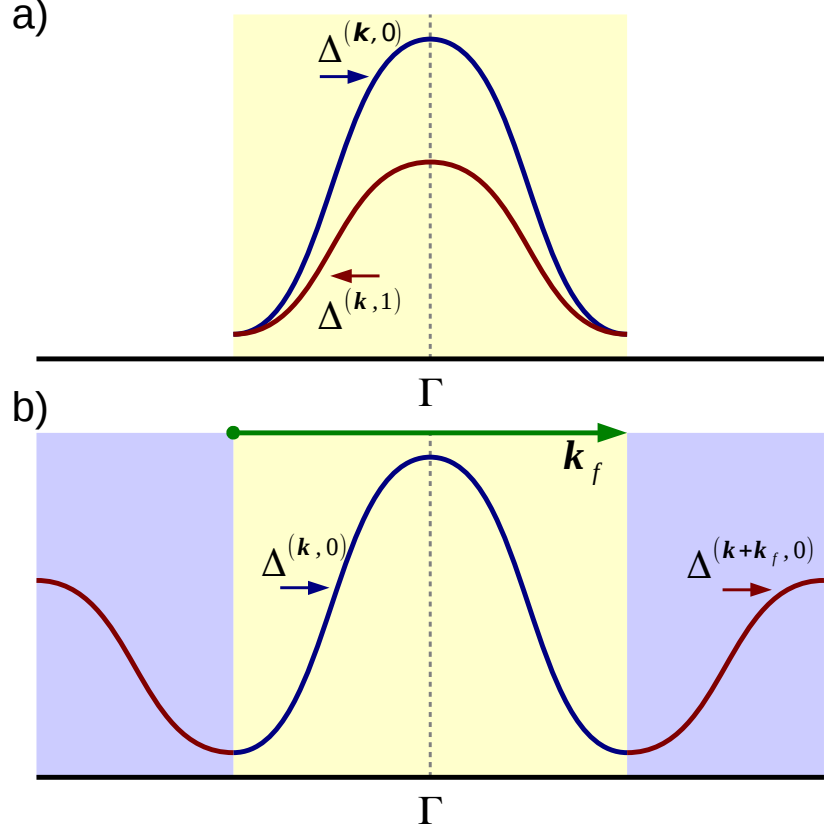

FIG. 1: Brillouin zone unfolding. (a) Folded bandstructure showing two bands belonging to two irreducible representations $\Delta^{(\boldsymbol{k}, 0)}$ and $\Delta^{(\boldsymbol{k}, 1)}$ of $\mathbb{S}$. (b) The unfolded bandstructure shows that $\Delta^{(\boldsymbol{k}, 1)}=\Delta^{\left(\boldsymbol{k}+\boldsymbol{k}_{f}, 0\right)}$, leading to the unfolded Brillouin zone, shown in yellow and purple backgrounds. The folding vector $\boldsymbol{k}_{f}$ is shown in green color.

more complex, and more sophisticated methods, such as Herring's method, are needed [14, 15]. We will just assume that $\boldsymbol{k}$ never lies on the BZ boundary, but it can be arbitrarily close to it. In cases where $\hat{U}_{i}=1$, i.e. where the operators $\hat{C}_{i}$ are just fractional translations, which is the case of translational unfolding, the little group $\mathbb{L}_{\boldsymbol{k}}$ will contain all fractional translations and will be the same for every $\boldsymbol{k}$. In this case, the irreducible representations of the little group $\Lambda^{(\boldsymbol{k}, \alpha)}$, are taken to be the irreducible representations of the group of fractional translations, modulo $\mathbb{T}$, meaning that two fractional translations are considered to be identical if they differ by $\boldsymbol{R}_{\boldsymbol{n}}$.

When $\boldsymbol{k}$ is invariant (up to the reciprocal lattice vector $\boldsymbol{K}$ ) under all point group operators of $\mathbb{C}$, then from the irreducible representation $\Delta^{(\boldsymbol{k})}$ of $\mathbb{T}, K$ one-dimensional irreducible representations $\Delta^{(\boldsymbol{k}, \alpha)}$ will be induced. When this is the case, the $\Lambda^{(\boldsymbol{k}, \alpha)}\left(\hat{U}_{i}\right)$ will be roots of unity so that we can write, in general,

$$
\Delta^{(\boldsymbol{k}, \alpha)}\left(\left(\hat{U}_{i} \mid \boldsymbol{\tau}_{i}\right)\right)=\exp \left(-2 \pi \mathrm{i} f_{i}^{\alpha} / K\right) \exp \left(-\mathrm{i} \boldsymbol{k} \cdot \boldsymbol{\tau}_{i}\right)
$$

where $f_{i}^{\alpha}$ is an integer such that $0 \leq f_{i}^{\alpha}<K$. The integers $f_{i}^{\alpha}$, with the operation of addition modulo $K$, constitute a group isomorphic to $\mathbb{L}_{\boldsymbol{k}}$. We use the convention that $\alpha=0$ denotes the unit irreducible representation, thus $f_{i}^{0}=0$. When (4) is taken into account, the 
projectors onto the irreducible subspaces are given by

$$
\hat{P}_{\boldsymbol{k} \alpha}=\frac{1}{\sqrt{K N}} \sum_{\boldsymbol{n}} \sum_{i} \exp \left(\mathrm{i} \boldsymbol{k} \cdot \boldsymbol{R}_{\boldsymbol{n}}\right)\left[\Delta^{(\boldsymbol{k}, \alpha)}\left(\hat{C}_{i}\right)\right]^{\dagger} \hat{S}_{\boldsymbol{n} i}
$$

so that the Bloch basis (2) corresponding to $\mathbb{S}$ can be written, in analogy to (1), as

$$
|\boldsymbol{k}, \mu, \alpha\rangle=\frac{1}{\sqrt{K N}} \sum_{\boldsymbol{n}} \sum_{i} \sum_{\nu} \exp \left(2 \pi \mathrm{i} f_{i}^{\alpha} / K\right) \exp \left(\mathrm{i} \boldsymbol{k} \cdot\left(\boldsymbol{R}_{\boldsymbol{n}}+\boldsymbol{\tau}_{i}\right)\right)\left|\boldsymbol{R}_{\boldsymbol{n}}, \nu\right\rangle W_{\nu \mu}\left(\hat{C}_{i}\right)
$$

By employing the basis Eq. (5), $\hat{H}_{\boldsymbol{k}}$ can be brought into block-diagonal form with $F$ blocks $\hat{H}_{\boldsymbol{k} \alpha}$ of size $P / K \times P / K$. Each block $\hat{H}_{\boldsymbol{k} \alpha}$ can be diagonalized separately and will yield $P / K$ bands. It is important to note that because of the addition of a fractional translation in Eq. (5), the reciprocal space period of blocks $\hat{H}_{\boldsymbol{k} \alpha}$ is larger than the BZ. Since the only difference between the blocks $\hat{H}_{\boldsymbol{k} \alpha}$ is in the exponential prefactor $\exp \left(-2 \pi \mathrm{i} f_{i}^{\alpha} / K\right)$ in (5), we can restrict ourselves to only one block, i. e. $\hat{H}_{\boldsymbol{k} \alpha}$, and then reproduce the other blocks, by allowing $\boldsymbol{k}$ to leave the BZ, because we can choose $\boldsymbol{k}_{f}^{\alpha \beta} \in \mathcal{B}(\mathcal{L})$, such that

$$
\begin{aligned}
\boldsymbol{k}_{f}^{\alpha \beta} \cdot \boldsymbol{R}_{\boldsymbol{n}} & =2 \pi z \\
\boldsymbol{k}_{f}^{\alpha \beta} \cdot \boldsymbol{\tau}_{i} & =2 \pi \frac{f_{i}^{\beta}-f_{i}^{\alpha}}{K}
\end{aligned}
$$

where $z$ is an integer. In this way, we can have $\hat{H}_{\boldsymbol{k} \beta}=$ $\hat{H}_{\boldsymbol{k}+\boldsymbol{k}_{f}^{\alpha \beta}, \alpha}$. The vectors $\boldsymbol{k}_{f}^{\alpha \beta}$ are the folding vectors. The diagonalization of the block $\hat{H}_{\boldsymbol{k} \alpha}$ and its extensions outside of the BZ, by the folding vectors, produces the unfolded bands. The BZ, enlarged by the $K$ folding vectors $\boldsymbol{k}_{f}^{\alpha \beta}$ is the unfolded BZ (Fig. 1 ).

It is important to stress here, that due to the fact that $\hat{C}_{i}$ involve the fractional translations $\boldsymbol{\tau}_{i}$, and that due to the requirement of the one-dimensionality of the irreducible representations of $\mathbb{S}$, the unfolded bandstructure represents the bandstructure of the crystal lattice with the unit cell size reduced by a factor of $K$, which can be simply translationally folded back along the folding vectors to represent the starting bandstructure, regardless of the point group operations $\hat{U}_{i}$. Because of this, we can effectively describe the electronic structure with a Hamiltonian of smaller dimensionality. We can also understand this in a different way. Since $|\boldsymbol{k}, \mu, \alpha\rangle$ are the symmetry adapted basis vectors, no interaction contained in the Hamiltonian can cause a transition between the states with different $\alpha$, thereafter all dynamical processes are contained within their respective irreducible subspaces. We have seen that different irreducible representations become equivalent if shifted by the folding vectors, meaning that no information is lost if we just keep a single irreducible representation, as long as we expand it onto the entire unfolded Brillouin zone.
One more important issue to note is that our requirement for the one-dimensional irreducible representations of $\mathbb{S}$, implies that the unfolding which utilizes the point group operations is exact only for $\boldsymbol{k}$ values which are invariant under all point group operations $\hat{U}_{i}$. For example, if we use the screw-axis operations, the unfolding will be exact only along a corresponding high-symmetry line in the Brillouin zone. However, if the electronic properties are dominantly one dimensional along the given highsymmetry line, the unfolding can still be used across the entire Brillouin zone, while preserving the accuracy up to a significant degree.

To unfold the bandstructure given in the Bloch basis (2), we just need to calculate the matrix elements of the projectors $\hat{P}_{\boldsymbol{k} \alpha}$. The matrix elements are given by

$$
\begin{aligned}
& {\left[\hat{P}_{\boldsymbol{k} \alpha}\right]_{\gamma \delta}=\left\langle\boldsymbol{k}, \gamma\left|\hat{P}_{\boldsymbol{k} \alpha}\right| \boldsymbol{k}, \delta\right\rangle} \\
& \quad=\frac{1}{K} \sum_{i} \exp \left(2 \pi \mathrm{i} \alpha f_{i}^{\alpha} / K\right) \exp \left(\mathrm{i} \boldsymbol{k} \cdot \boldsymbol{\tau}_{i}\right) W_{\gamma \delta}\left(\hat{C}_{i}\right)
\end{aligned}
$$

If we assume that from the bandstructure calculations we obtain bands $|\boldsymbol{k}, n\rangle$, where $n$ is the band index, we can unfold the bands by applying the projectors (7) to the column-vector containing the projections of bands onto the localized states $w_{\boldsymbol{k}, n}^{\mu}=\langle\mathbf{0}, \mu \mid \boldsymbol{k}, n\rangle$. In our particular case, we have used the Vienna Ab-Initio Simulations Package (VASP) to obtain the bandstructure. Since, within the VASP package, the exponential factors $\exp \left(-i \boldsymbol{k} \cdot \boldsymbol{s}_{\mu}\right)$ for fractional site vector $\boldsymbol{s}_{\mu}$ are already included in the projections $w_{\boldsymbol{k}, n}^{\mu}$, the exponential factors in Eq. (7) can be omitted, simplifying the expressions even further.

\section{Unfolding of tight-binding models}

In general, any observable $\hat{A}_{\boldsymbol{k}}$ can be unfolded by employing the projectors $\hat{P}_{\boldsymbol{k} \alpha}$ so that $\hat{A}_{\boldsymbol{k} \alpha}=\hat{P}_{\boldsymbol{k} \alpha} \hat{A}_{\boldsymbol{k}} \hat{P}_{\boldsymbol{k} \alpha}$.

With the help of Eq. (5) it is also possible to unfold tight-binding models. The matrix elements of the Hamiltonian in the tight-binding model are defined in the Bloch 
basis Eq. (2) as

$$
\begin{aligned}
{\left[\hat{H}_{\boldsymbol{k}}\right]_{\mu \nu} } & =\left\langle\mathbf{0}, \mu\left|\hat{H} \hat{P}_{\boldsymbol{k}}\right| \mathbf{0}, \nu\right\rangle \\
& =\sum_{\boldsymbol{R}_{\boldsymbol{n}}} t_{\mu \nu}\left(\boldsymbol{R}_{\boldsymbol{n}}\right) \exp \left(\mathrm{i} \boldsymbol{k} \cdot \boldsymbol{R}_{\boldsymbol{n}}\right)
\end{aligned}
$$

where $t_{\mu \nu}\left(\boldsymbol{R}_{\boldsymbol{n}}\right)=\left\langle\mathbf{0}, \mu|\hat{H}| \boldsymbol{R}_{\boldsymbol{n}}, \nu\right\rangle$ are the hopping energies. This result follows from $\hat{H}_{\boldsymbol{k}}=\hat{P}_{\boldsymbol{k}} \hat{H} \hat{P}_{\boldsymbol{k}}$ and the facts that $\hat{H}$ commutes with $\hat{P}_{\boldsymbol{k}}$ and that $\hat{P}_{\boldsymbol{k}}$ is idempotent. We define the matrix composed of the hopping energies $t_{\mu \nu}\left(\boldsymbol{R}_{\boldsymbol{n}}\right)$ as $\hat{t}\left(\boldsymbol{R}_{\boldsymbol{n}}\right)$. The unfolding of the tight-binding model is achieved by calculating the matrix elements of the Hamiltonian in the basis Eq. (5) and then casting the resulting expression in the form of Eq. (8)

$$
\begin{aligned}
{\left[\hat{H}_{\boldsymbol{k} \alpha}\right]_{\mu \nu} } & =\left\langle\mathbf{0}, \mu\left|\hat{H} \hat{P}_{\boldsymbol{k} \alpha}\right| \mathbf{0}, \nu\right\rangle \\
& =\sum_{\boldsymbol{n}} \sum_{i} t_{\mu \nu}^{\alpha}\left(\boldsymbol{R}_{\boldsymbol{n}}+\boldsymbol{\tau}_{i}\right) \exp \left(\mathrm{i} \boldsymbol{k} \cdot\left(\boldsymbol{R}_{\boldsymbol{n}}+\boldsymbol{\tau}_{i}\right)\right)
\end{aligned}
$$

where the corresponding hopping energy matrix is $\hat{t}^{\alpha}\left(\boldsymbol{R}_{\boldsymbol{n}}+\boldsymbol{\tau}_{i}\right)$. The hopping energies of the unfolded TB model can then be read off as coefficients of the exponential terms $\exp \left(\mathrm{i} \boldsymbol{k} \cdot\left(\boldsymbol{R}_{\boldsymbol{n}}+\boldsymbol{\tau}_{i}\right)\right)$. The hopping energy matrices $\hat{t}^{\alpha}\left(\boldsymbol{R}_{\boldsymbol{n}}+\boldsymbol{\tau}_{i}\right)$ will have the same block-diagonal structure of $\hat{H}_{\boldsymbol{k} \alpha}$. The general expression for the hopping energies is then

$$
\hat{t}^{\alpha}\left(\boldsymbol{R}_{\boldsymbol{n}}+\boldsymbol{\tau}_{i}\right)=\frac{\exp \left(2 \pi \mathrm{i} f_{i}^{\alpha} / K\right)}{K} \hat{t}\left(\boldsymbol{R}_{\boldsymbol{n}}\right) \hat{W}\left(\hat{C}_{i}\right)
$$

It should be noted here that for $K>2$, the unfolded hopping energies can become complex due to the prefactor $\exp \left(2 \pi \mathrm{i} f_{i}^{\alpha} / K\right)$. However, this prefactor does not affect the eigenvalues and eigenvectors of $\hat{H}_{\boldsymbol{k} \alpha}$ since it amounts to an overall, $\boldsymbol{k}$-independent, unitary transformation of the Hamiltonian. Furthermore, in the unfolded picture, we extend a single irreducible representation beyond the BZ boundaries, so that in the tight-binding model we expect to have a single set of hopping energies independent of the irreducible representation. This manifestly does not hold for Eq. 10p since the exponential prefactor depends on $\alpha$. Because of this, the exponential prefactor in Eq. (10) can be dropped and the irreducible representation-independent unfolded hopping energies can be defined as

$$
\hat{t}\left(\boldsymbol{R}_{\boldsymbol{n}}+\boldsymbol{\tau}_{i}\right)=\frac{1}{K} \hat{t}\left(\boldsymbol{R}_{\boldsymbol{n}}\right) \hat{W}\left(\hat{C}_{i}\right)
$$

When the unfolded hopping energies are defined in this way, the index $\alpha$ can be omitted from Eq. (9). Since we already concluded that the $\hat{t}^{\alpha}\left(\boldsymbol{R}_{\boldsymbol{n}}+\boldsymbol{\tau}_{i}\right)$ are blockdiagonal, a reduction of the dimensionality of the tightbinding model is achieved. Practically, this means that orbital indices $\mu$ and $\nu$ can be taken to run only over the first block in the block-diagonalized Hamiltonian, since other blocks are symmetrically equivalent.

\section{Relation to ARPES}

Angle resolved photoemission is one of the most direct ways to experimentally observe the bandstructure in solids [16. However, the interpretation of raw experimental data is a very complicated process and often relies heavily on comparisons with density functional theory calculations. This becomes especially difficult in systems with broken translational symmetry, since, on the one hand, supercell calculations have to be employed by density functional theory resulting in complicated folded bandstructures, while on the other hand ARPES data often shows the unfolded bandstructure, sometimes offset away from the first Brillouin zone [6, 17. This was already discussed in the context of bandstructure unfolding [6]. However, some important issues were not considered, like the fact that multiple irreducible representations are involved in the unfolding as well as the effect of high symmetry elements of the Brillouin zone.

The observed photoelectron intensity at energy $\omega$ in ARPES experiments can be directly related to the oneelectron spectral function [18, 19]

$$
I(\omega) \sim \sum_{\boldsymbol{k}} \sum_{f} \sum_{i j} M_{\boldsymbol{k} f i} A_{\boldsymbol{k} i j}(\omega) M_{\boldsymbol{k} j f}
$$

where $M_{\boldsymbol{k} f i}=\langle\boldsymbol{k}, f|\boldsymbol{\varepsilon} \cdot \hat{\boldsymbol{x}}| \boldsymbol{k}, i\rangle$ are the dipole matrix elements between the initial state $|\boldsymbol{k}, i\rangle$ and the final state $|\boldsymbol{k}, f\rangle$ and $\hat{A}_{\boldsymbol{k} i j}(\omega)$ are the matrix elements of the oneelectron spectral function. Using the projectors from Eq. (1) this can be rewritten as

$$
I(\omega) \sim \operatorname{tr}\left(\sum_{\boldsymbol{k}} \varepsilon \cdot \hat{\boldsymbol{x}} \hat{P}_{\boldsymbol{k}} \hat{A}(\omega) \hat{P}_{\boldsymbol{k}} \varepsilon \cdot \hat{\boldsymbol{x}}\right)
$$

where the trace is taken only over the final states and $\hat{A}$ is restricted to the occupied subspace. If the observed system has approximate symmetry given by a group $\mathbb{S} \triangleright \mathbb{T}$, either because (i) we have a slight breaking of the translational symmetry, or (ii) most of the contribution to the sum over $\boldsymbol{k}$ in Eq. (12) comes from the surface states lying in the high-symmetry element of the Brillouin zone, we can extend the summation over $\boldsymbol{k}$ onto the irreducible subspaces of $\mathbb{S}$ so that we have

$$
I(\omega) \sim \operatorname{tr}\left(\sum_{\boldsymbol{k}} \sum_{\alpha \beta} \varepsilon \cdot \hat{\boldsymbol{x}} \hat{P}_{\boldsymbol{k} \alpha} \hat{A}(\omega) \hat{P}_{\boldsymbol{k} \beta} \varepsilon \cdot \hat{\boldsymbol{x}}\right)
$$

Since $\mathbb{S}$ is an approximate symmetry, the off-diagonal blocks $\hat{P}_{\boldsymbol{k} \alpha} \hat{A} \hat{P}_{\boldsymbol{k} \beta}$ can be neglected. In addition, we can use the folding relations, Eq. (6) to replace the summation over the irreducible representation index $\alpha$ with the summation over the unfolded Brillouin zone to obtain

$$
I(\omega) \sim \operatorname{tr}\left(\sum_{\boldsymbol{k}_{\alpha}} \varepsilon \cdot \hat{\boldsymbol{x}} \hat{P}_{\boldsymbol{k} \alpha} \hat{A}(\omega) \hat{P}_{\boldsymbol{k} \alpha} \varepsilon \cdot \hat{\boldsymbol{x}}\right)
$$


where $\boldsymbol{k}_{\alpha}=\boldsymbol{k}+\boldsymbol{k}_{f}^{\alpha \beta}, \forall \beta$, belong to the Brillouin zone unfolded in accordance with Eq. (6). In this case, the ARPES experiment will observe the spectral function $\hat{A}_{\boldsymbol{k} \alpha}(\omega)=\hat{P}_{\boldsymbol{k} \alpha} \hat{A}(\omega) \hat{P}_{\boldsymbol{k} \alpha}$ instead of the spectral function $\hat{A}_{\boldsymbol{k}}(\omega)=\hat{P}_{\boldsymbol{k}} \hat{A}(\omega) \hat{P}_{\boldsymbol{k}}$. One important detail in Eq. (13) is the fact that the irreducible representation $\alpha$ is only selected through the effect of dipole operators and the trace over the final states. This means, that the actual irreducible representation observed in the experiment depends on the experimental conditions.

This is a very important conclusion, since it tells us that in order to carefully interpret raw ARPES data, we have to take into account two considerations. The first one is the possible effect of high symmetry elements in the Brillouin zone. This implies the incorporation of point group operations into the unfolding - we would like to stress here, that this issue is distinct from orbital symmetry selection by means of polarization. The second consideration is that we have to take into account all irreducible representations arising from the unfolding process and then use Eq. (6) to reconstruct the bandstructure from the ARPES data, if necessary.

\section{APPLICATIONS}

Iron-based superconductors provide an excellent playground for the unfolding method presented here. The crystal structures of iron pnictides and iron chalcogenides consist of layers of Fe atoms tetrahedrally coordinated by the pnictogen/chalcogen atoms as shown in Fig. 2 (a). The various compounds may show differences in the stacking sequence of the iron pnictogen/chalcogen layers, as well as in the composition of the spacer layers. For most of the iron pnictide/chalcogenide families, a minimal translationally invariant unit cell consists of two iron and two pnictogen/chalcogen atoms. This unit cell can further be reduced by considering the glide-mirror operations, which combine the translations between the nearest-neighbor iron atoms with reflections in the $x y$ plane, thus mapping two translationally inequivalent iron and pnictogen/chalcogen sites into each other.

In the following we shall consider four representative unfolding examples. In the first case we will apply translational unfolding on a $2 \times 1$ supercell of FeSe where the translational symmetry is kept in the supercell. In the second case we will apply translational unfolding on a P-doped $\mathrm{CaFe}_{2} \mathrm{As}_{2} 2 \times 2$ supercell $\left(\mathrm{Ca}_{4} \mathrm{Fe}_{8} \mathrm{As}_{7} \mathrm{P}\right)$ where the translational symmetry has been broken by the substitution of one As by P. In the third case we unfold the 16-band tight-binding model $(2 \times 5 \mathrm{Fe}$ bands and $2 \times 3$ Se bands) for FeSe at $10 \mathrm{GPa}$ to an 8-band tight-binding model. At this pressure, the structure shows important dispersion along $k_{z}$ and it allows for an analysis of the unfolding procedure in all three directions. Finally in the fourth case we apply unfolding of the two-iron unit cell to

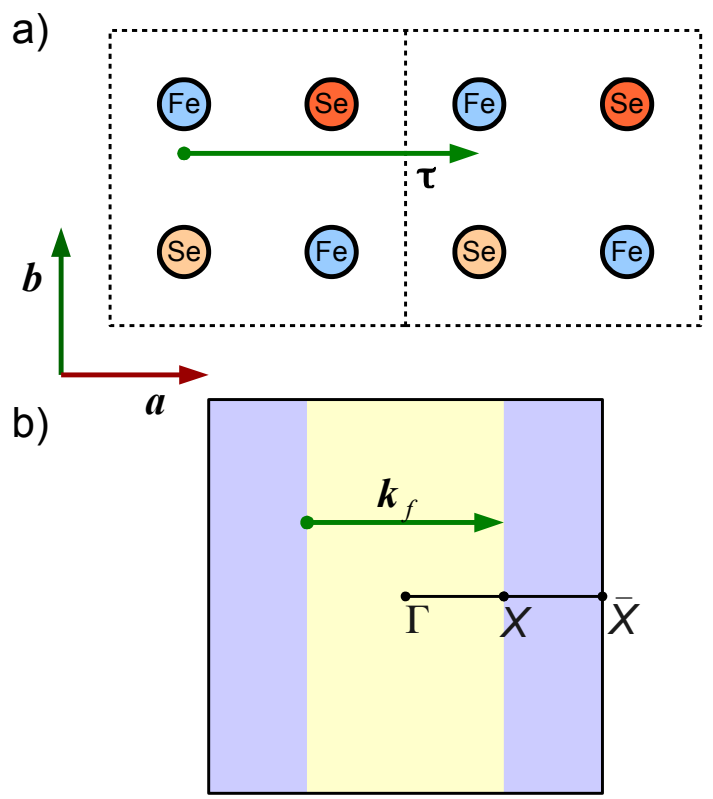

FIG. 2: (a) Projection on the $a b$-plane of a $2 \times 1$ supercell of tetragonal FeSe. The fractional translation $\boldsymbol{\tau}$ along $\boldsymbol{a}$ is shown in green color. The iron atoms lie in the same plane, parallel to the plane of the drawing, while the lighter colored selenium atoms are vertically displaced above the plane, and the darker colored ones are below. (b) $k_{z}=0$ plane of the Brillouin zone corresponding to the single unit cell of FeSe. The yellow filling marks the Brillouin zone corresponding to the supercell. The folding vector $\boldsymbol{k}_{f}$ is shown in green color.

the one-iron unit cell representation in the specific case of the body centered space group $I 4 / \mathrm{mmm}$. With this last example we want to show that the unfolding procedure is independent of whether the space group is body centered or not.

\section{Translational unfolding of FeSe}

As a first example, we consider a simple case of translational unfolding, where the translation group $\mathbb{T}$ is expanded by fractional translations $\hat{C}_{i}=\left(1 \mid \boldsymbol{\tau}_{i}\right)$. We define a $2 \times 1$ supercell of tetragonal FeSe (Fig. 2 (a)) by doubling the unit cell along the $\boldsymbol{a}$ axis of FeSe. In Fig. 3 (a) we show the corresponding supercell bandstructure along the path $\Gamma-X$ (Fig. 2(b)). In order to unfold the bands, we employ the fractional translation $\boldsymbol{\tau}=\boldsymbol{a}$ which is an additional symmetry that the supercell has on top of the translational symmetry $\mathbb{T}$. With this, the factor group $\mathbb{C}$ is isomorphic to the cyclic group of order two, with the generator $(1 \mid \boldsymbol{\tau})$ and from every irreducible representation $\mathbb{T}$, two irreducible representations with $\alpha=0$ and $\alpha=1$ are induced, so that the generator Eq. (4) is represented 


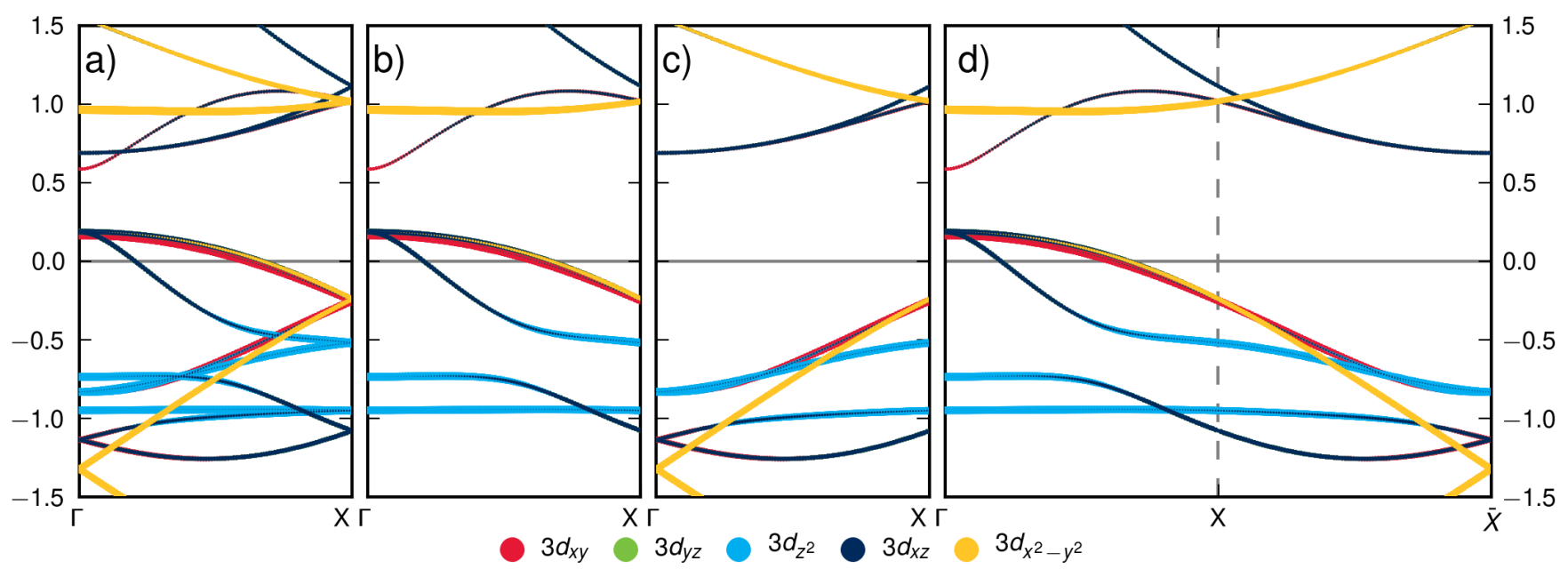

FIG. 3: Unfolding of the tetragonal FeSe doubled along the $\boldsymbol{a}$-axis. (a) The folded bandstructure. (b) Projection of bands onto $\Delta^{(\boldsymbol{k}, 0)}$. (c) Projection of bands onto $\Delta^{(\boldsymbol{k}, 0)}$. (d) Unfolded picture. Irreducible representation $\Delta^{(\boldsymbol{k}, 0)}$ is extended past the boundaries of the Brillouin zone by the folding vector $\boldsymbol{k}_{f}=(\pi / a, 0,0)$.

by

$$
\begin{aligned}
& \Delta^{(\boldsymbol{k}, 0)}((1 \mid \boldsymbol{\tau}))=\exp (-\mathrm{i} \boldsymbol{k} \cdot \boldsymbol{\tau}) \\
& \Delta^{(\boldsymbol{k}, 1)}((1 \mid \boldsymbol{\tau}))=-\exp (-\mathrm{i} \boldsymbol{k} \cdot \boldsymbol{\tau})
\end{aligned}
$$

The resulting band projections onto the irreducible representations are shown in Fig. 3 (b) and (c) while the unfolded picture, where the irreducible representation $\Delta^{(k, 0)}$ is extended outside the supercell BZ is shown in Fig. 3 (d). Evidently $\Delta^{\left(\boldsymbol{k}+\boldsymbol{k}_{f}, 0\right)}=\Delta^{(\boldsymbol{k}, 1)}$, with $\boldsymbol{k}_{f}=(\pi / a, 0,0)$.

\section{Translational unfolding of P-doped $\mathrm{CaFe}_{2} \mathrm{As}_{2}$}

The unfolding shown on Fig. 3 is perfect, because the fractional translation $(1 \mid \boldsymbol{\tau})$ is an exact symmetry of the supercell and every band will belong to only one of the irreducible representations of $\mathbb{S}$. In a more realistic case, where the operations $\hat{C}_{i}$ are only approximate symmetries, the bands will have nonzero projections onto multiple irreducible representations, although usually, one of the irreducible representations will be dominantly present in every band. Such a situation occurs, for example, when studying doped compounds. Here we have chosen to investigate the phosphorus doped $\mathrm{CaFe}_{2} \mathrm{As}_{2}$.

It is well known that when pressure is applied on $\mathrm{CaFe}_{2} \mathrm{As}_{2}$, it undergoes a magneto-structural phase transition from a magnetically ordered orthorhombic phase to a non-magnetic, collapsed tetragonal phase 20, 21. In previous studies 22 24, we simulated the application of pressure under different conditions by means of density functional theory calculations and were able to predict the appearance of the collapsed tetragonal phase at a critical pressure which is accompanied by the disappearance of the Fermi surface pockets centered around the $\Gamma$ point. This feature has been recently confirmed by angle resolved photoemission experiments [25, 26].

An orthorhombic to collapsed tetragonal phase transition in $\mathrm{CaFe}_{2} \mathrm{As}_{2}$ can also be induced by chemical pressure. For example, substitutional doping of phosphorus into the arsenic sites causes $\mathrm{CaFe}_{2} \mathrm{As}_{2}$ to enter the collapsed tetragonal phase at a doping level of around $5 \%$ 27. In order to fully understand how chemical pressure is related to the application of physical pressure, we have performed a sequence of full structural relaxations of $\mathrm{P}$-doped $\mathrm{CaFe}_{2} \mathrm{As}_{2}$. For the different doping levels we have considered supercells of various sizes. Our density functional theory calculations predict that P-doped $\mathrm{CaFe}_{2} \mathrm{As}_{2}$ undergoes an orthorhombic to collapsed tetragonal phase transition for a doping between $9.375 \%$ and $12.5 \%$ in good agreement with the experimental observations [27. In order to analyze the electronic structure in the collapsed tetragonal phase of $\mathrm{P}$ doped $\mathrm{CaFe}_{2} \mathrm{As}_{2}$, we have to perform the unfolding of the bandstructure.

Relaxed $\mathrm{Ca}\left(\mathrm{FeAs}_{0.875} \mathrm{P}_{0.125}\right)_{2}$ has an orthorhombic unit cell where the phosphorus atom is breaking the translational symmetry as shown in Fig. 4. The unit cell of $\mathrm{Ca}\left(\mathrm{FeAs}_{0.875} \mathrm{P}_{0.125}\right)_{2}$ is a supercell consisting of four primitive unit cells of $\mathrm{CaFe}_{2} \mathrm{As}_{2}$. This unit cell contains a total of eight iron atoms. The corresponding fractional translations are $\boldsymbol{\tau}_{1}=(\boldsymbol{a}+\boldsymbol{b}) / 2, \boldsymbol{\tau}_{2}=(\boldsymbol{a}+\boldsymbol{c}) / 2$ and $\boldsymbol{\tau}_{3}=\boldsymbol{\tau}_{1}+\boldsymbol{\tau}_{2}$, where $\boldsymbol{a}, \boldsymbol{b}$ and $\boldsymbol{c}$ are the unit vectors of the supercell, as shown in Fig. 4. These fractional translations map the two translationally inequivalent iron atoms to the eight iron atoms of the supercell.

The corresponding bandstructure is shown in Fig. 5 


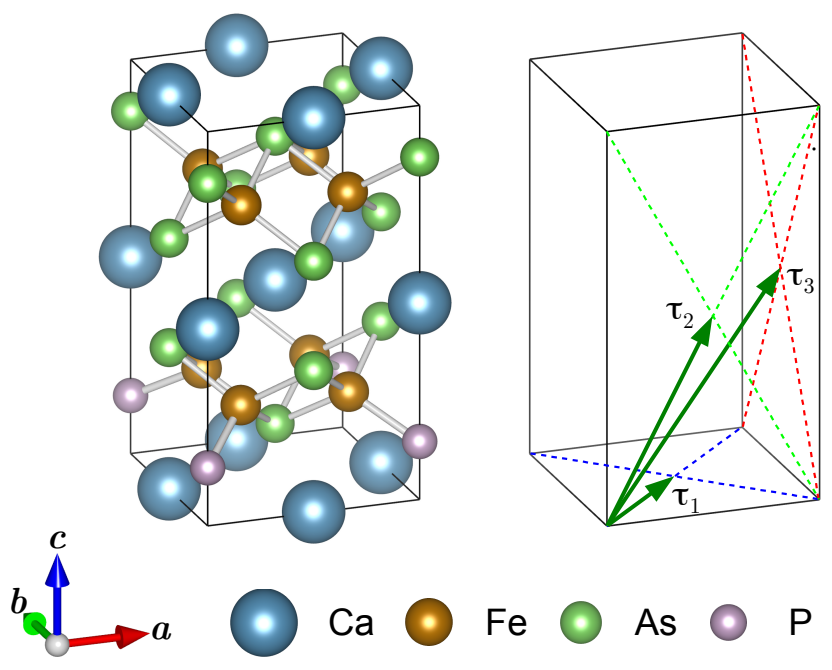

FIG. 4: The unit cell of $\mathrm{Ca}\left(\mathrm{FeAs}_{0.875} \mathrm{P}_{0.125}\right)_{2}$. The fractional translations $\boldsymbol{\tau}_{i}$ are shown in green color.

(a). The bandstructure is calculated along the path given by $(0,0,0)-(2 \pi / a, 0,0)-(2 \pi / a, 2 \pi / a, 0)-(0,0,0)-$ $(0,0,2 \pi / c)$. Four irreducible representations can be induced. We will select the irreducible representation $\Delta^{(k, 0)}$ and extend it to the unfolded Brillouin zone. The resulting unfolded bandstructure, obtained by extending the irreducible representation $\Delta^{(\boldsymbol{k}, 0)}$ is shown in Fig. 5 (b). Despite the fact that the fractional translations $\boldsymbol{\tau}_{i}$ are not the exact symmetries of $\mathrm{Ca}\left(\mathrm{FeAs}_{0.875} \mathrm{P}_{0.125}\right)_{2}$, the band projections onto different irreducible representations are still mostly orthogonal, having relatively clean unfolded bands as a result. This allows us to clearly see the disappearance of the hole pockets centered around $\Gamma$, since the set of three hole $t_{2 g}$ bands is pushed below the Fermi level by around $0.2 \mathrm{eV}$. Comparison of the unfolded bands to the bandstructure of the collapsed tetragonal phase of $\mathrm{CaFe}_{2} \mathrm{As}_{2}$ under pressure [23, 25] confirms that phosphorus doping and application of hydrostatic pressure affect the structural and electronic properties of $\mathrm{CaFe}_{2} \mathrm{As}_{2}$ in a remarkably similar way.

\section{Unfolding from a 16-band to an 8-band tight-binding model for FeSe under pressure}

We will now demonstrate the use of Eq. (10) and Eq. (11) for the unfolding of tight-binding models. We consider as a test system FeSe at $10 \mathrm{GPa}$ with significant dispersion in all three directions. The crystal structure has been obtained from ab-initio simulations of hydrostatic pressure at $10 \mathrm{GPa} 23,24$. We have used the projective Wannier functions as implemented in the FPLO code 28 for the derivation of the 16-band tight-binding model which consists of five $3 d$ orbitals per iron site, and three $4 p$ orbitals per selenium site. The structure under $10 \mathrm{GPa}$ of hydrostatic compression is chosen because the three dimensional character of the Fermi surface is more pronounced compared to ambient pressure.

As previously mentioned, the two translationally nonequivalent iron sites can be mapped onto each other with the help of the glide mirror operations $\hat{C}_{i}=\left(\boldsymbol{\tau}_{i} \mid \hat{\sigma}_{z}\right)$ with $i=1,2$. The fractional translations $\boldsymbol{\tau}_{i}$ connect the nearest neighbor iron atoms as shown in Fig 2 (a), while $\hat{\sigma}_{z}$ is a reflection in the $x y$-plane. To unfold we can choose one of the $\hat{C}_{i}$ operations and then induce the irreducible representations of $\mathbb{S}=\mathbb{T} \cup \mathbb{T} \hat{C}_{i}$. In accordance with Ref. 12, we call $\mathbb{S}$ the glide-mirror group. Since the factor group is of index two, two one-dimensional irreducible representations will be induced in the $k_{z}=0$ plane of the Brillouin zone. Because the electronic dispersion in FeSe is weaker along the $\boldsymbol{k}_{z}$ axis, we can expect that irreducible representations induced in the $k_{z}=0$ plane will give adequate unfolding across the rest of the Brillouin zone.

In these two irreducible representations, the glide mirror operation will have the same representation as did the fractional translation in Eq. 14. However, what differentiates the case of the glide-mirror unfolding from the purely translational unfolding is the orbitally selective action of the matrices $\hat{W}\left(\hat{C}_{i}\right)$ in Eq. (7). Namely, in the case of translational unfolding, matrices $\hat{W}\left(\hat{C}_{i}\right)$ act equally on all orbitals, while in the case of glide-mirror unfolding, they act differently, depending on whether the orbitals are symmetric or antisymmetric with respect to the reflections in the $x y$-plane. For example, the $3 d_{z^{2}}$ orbital will stay invariant, while $3 d_{x z}$ will pick up a minus sign under the action of $\hat{\sigma}_{z}$.

We have used Eq. 10 to create the two sets of hopping energies, corresponding to two induced irreducible representations. These two sets correspond to the same 8-band tight-binding model, up to the unitary transformation. The bandstructure calculated from the 16-band and two 8-band tight-binding models along the path in the $k_{z}=0$ plane of the Brillouin zone is shown in Fig. 6 (a). The corresponding Fermi surface slice in the $k_{z}=0$ plane is shown in Fig. 6 (c). In accordance with Eq. (6) and the electronic structure shown in Fig. 6 the folding vector is $\boldsymbol{k}_{f}^{01}=(\pi, \pi, 0)$ with respect to one iron Brillouin zone (as a convention, we will always specify folding vectors with respect to the unfolded Brillouin zone). It is evident that the unfolding to the 8-band model is perfect in the $k_{z}=0$ plane. Since the $k_{z}= \pm \pi / c$ planes are also the high-symmetry planes for the reflections in the $x y$-plane, the unfolding will be perfect there too. We can thus expect the largest deviations from the perfect unfolding around the $k_{z}= \pm \pi / 2 c$ plane. This can be seen in the bandstructure shown in Fig. 6 (b), taken along the path shown in Fig. 6 (a) shifted by $(0,0, \pi / 2 c)$. The deviations of the unfolded bands are evident. However, 


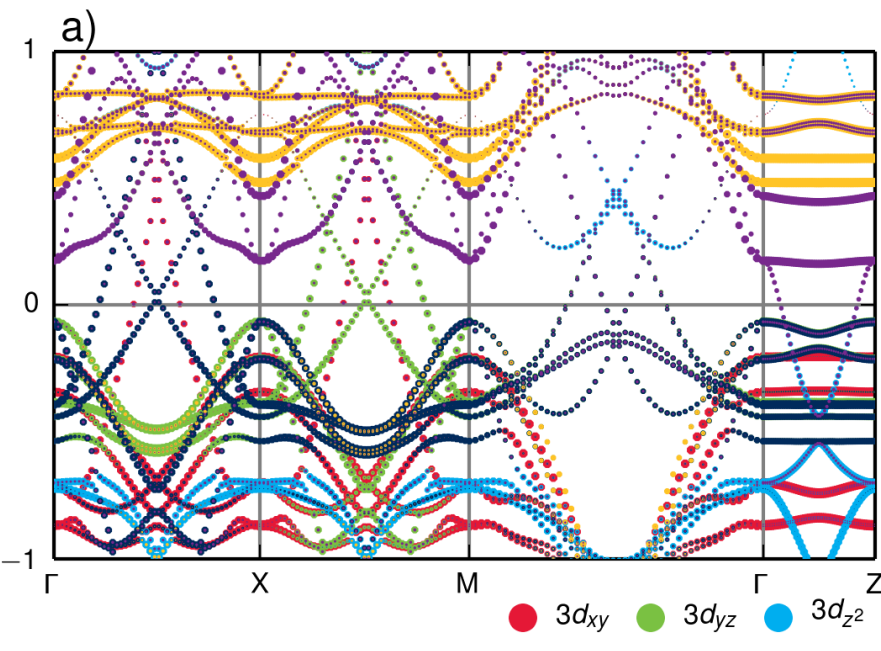

b)

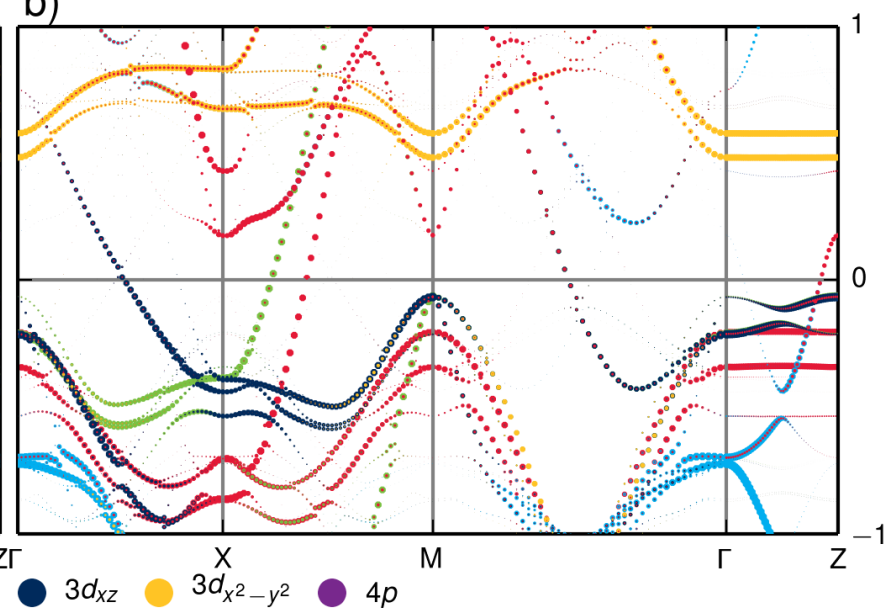

FIG. 5: Unfolding of $\mathrm{Ca}\left(\mathrm{FeAs}_{0.875} \mathrm{P}_{0.125}\right)_{2}$ bandstructure. (a) Bandstructure of the $\mathrm{Ca}\left(\mathrm{FeAs}_{0.875} \mathrm{P}_{0.125}\right)_{2}$ supercell. (b) The unfolded bandstructure obtained from $\Delta^{(\boldsymbol{k}, 0)}$.

the deviations of the top ten bands, which are the bands dominated by the $3 d$ orbital character, are much smaller than in the bands dominated by the $4 p$ orbital character. This is a consequence of the crystal structure; the iron atoms are stationary under the action of $\hat{\sigma}_{z}$, while the selenium atoms are not. Due to this property, the Fermi surface can be unfolded almost exactly across the entire Brillouin zone. The Fermi surface slice in the $k_{z}=\pi / 2 c$ is shown in Fig. 6(d), while the vertical slice in the $k_{y}=0$ plane is shown in Fig. 6 (e). It is remarkable that the full three dimensional structure of the innermost Fermi surface pocket, centered at $\Gamma$, is retained with high accuracy in the unfolded model, despite the fact that the underlying unfolding symmetry is purely two-dimensional.

\section{Unfolding the bandstructure of $\mathrm{CaFe}_{2} \mathrm{As}_{2}$ to the one-iron equivalent Brillouin zone}

Finally, we would like to point out that the glide-mirror group can also be used to unfold the bandstructures of iron-based superconductors with a centered unit cell, described by symmorphic space groups. In this particular example, we use the ambient pressure structure of $\mathrm{CaFe}_{2} \mathrm{As}_{2}$ measured at a temperature $T=250 \mathrm{~K}$ and described by the body-centered spacegroup $I 4 / \mathrm{mmm}$ [21]. $\mathrm{CaFe}_{2} \mathrm{As}_{2}$ was selected because it features additional electron Fermi surface pockets which make the one iron Fermi surface determination more complicated.

The glide-mirror operations as well as the irreducible representations are identical to the case of FeSe. The main difference here is that the resulting folding vector is $(\pi, \pi, \pi)$, corresponding to the unfolding onto the simple tetragonal one iron unit cell. This result is not immediately obvious, since we are only using the symmetry of the iron-arsenic layer, and it is to be expected, in the naïve picture, that the resulting unfolding would result in the one iron body-centered unit cell and the $(\pi, \pi, 0)$ folding vector. However, due to the body-centered symmetry the $(\pi, \pi, 0)$ folding vector relates $\Gamma$ and $Z$ points, as shown in Fig. 8 . In addition, unlike $(\pi, \pi, \pi)$, the $(\pi, \pi, 0)$ folding vector does not satisfy Eq. (6), which underlines their usefulness.

Fig. 7 (a) shows the bandstructure in the two-iron equivalent Brillouin zone, while Fig. 7 (b) shows the unfolded bandstructure in the one-iron equivalent Brillouin zone obtained by extending $\Delta^{(\boldsymbol{k}, 0)}$. Evidently, the unfolding remains perfect in the $k_{z}=0$ plane. However, this is no longer the case across the entire Brillouin zone. For instance, along the $\Gamma-Z$ path weak traces of bands from $\Delta^{(k, 1)}$ can be observed. In comparison to FeSe, in the vicinity of the Femi level, the $\mathrm{CaFe}_{2} \mathrm{As}_{2}$ bandstructure features more orbital weight of arsenic $4 p$ and some calcium $3 d$ character which makes unfolding outside of the high-symmetry plane less accurate. Nevertheless, we have enough information to clearly discern the topology of the unfolded Fermi surface. Figs. 7 (c)-(e) show the $k_{z}=0$ cut of the $\mathrm{CaFe}_{2} \mathrm{As}_{2}$ Fermi surface. Because of the body-centered arrangement of the Brillouin zones shown in Fig. 8 this is also the $k_{z}=\pi$ cut of the Fermi surface, offset by $(\pi, \pi, 0)$ and as a result the connectivity of the Fermi surface elements can be deduced between the $k_{z}=0$ and $k_{z}=\pi$ planes. 


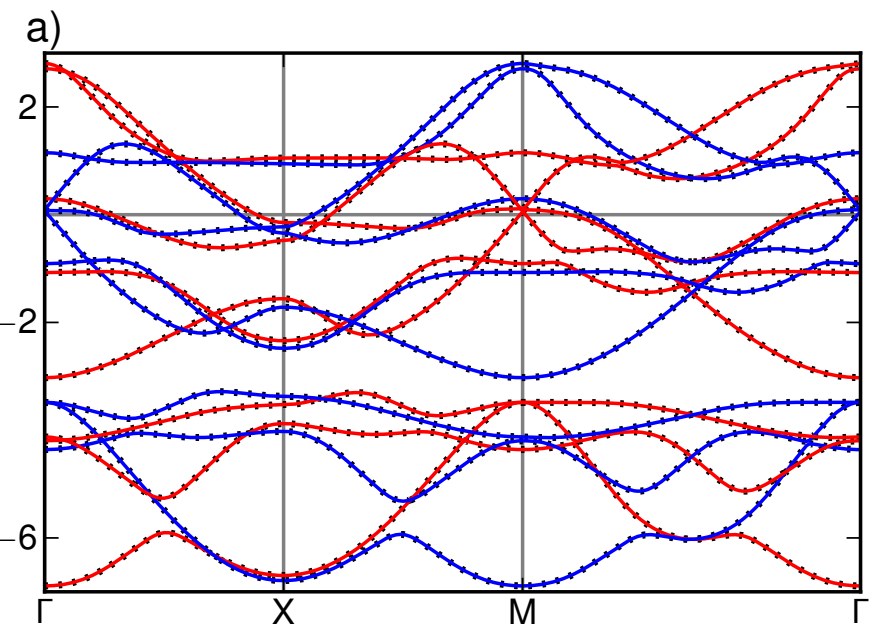

b)

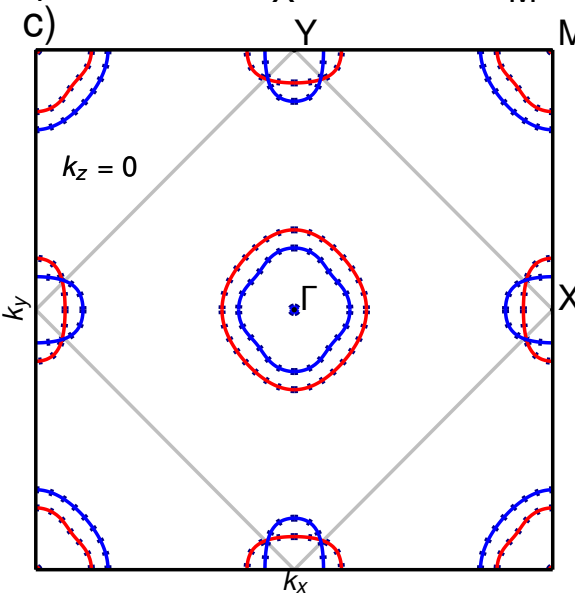

$\mathrm{M}$ d)

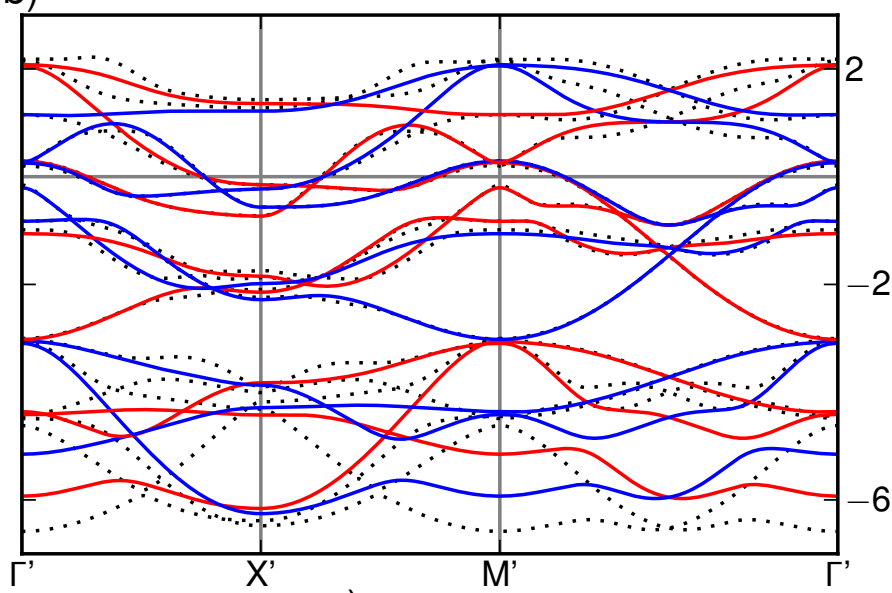

(d)

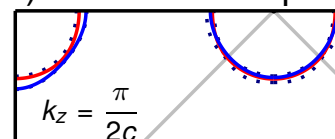

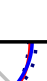

M' e)

A

\section{2} . 
a)

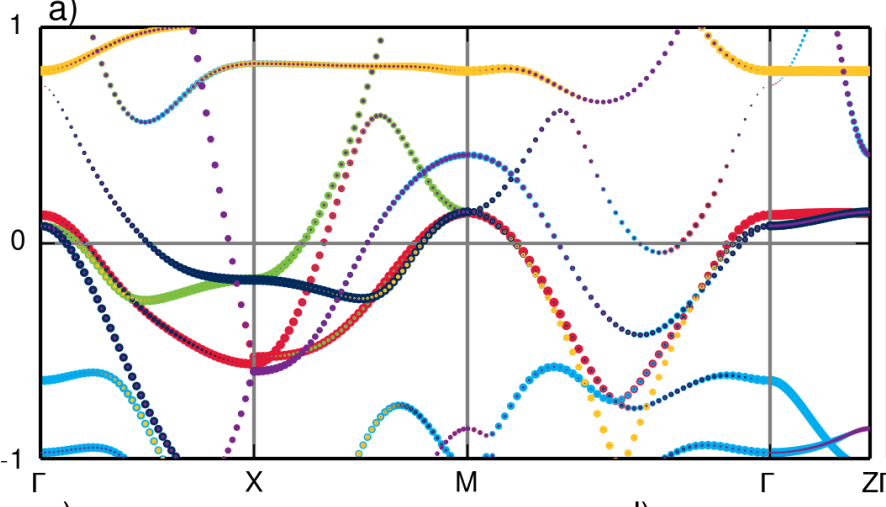

c)

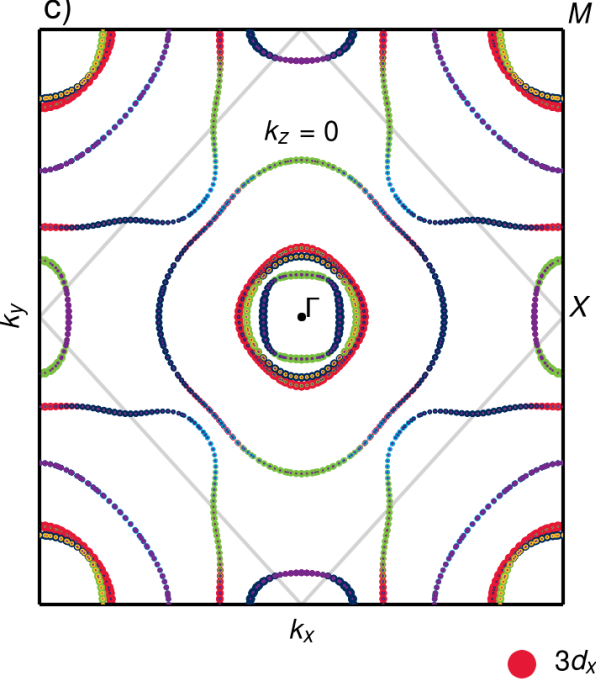

b)

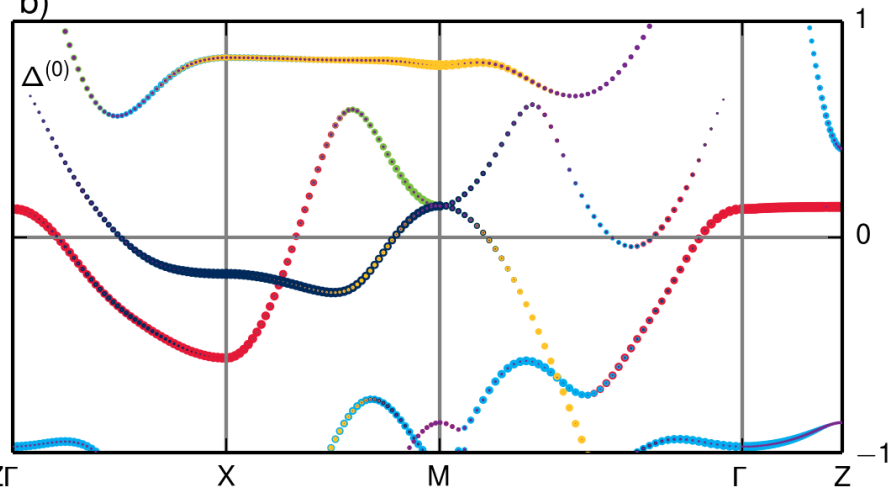

d)

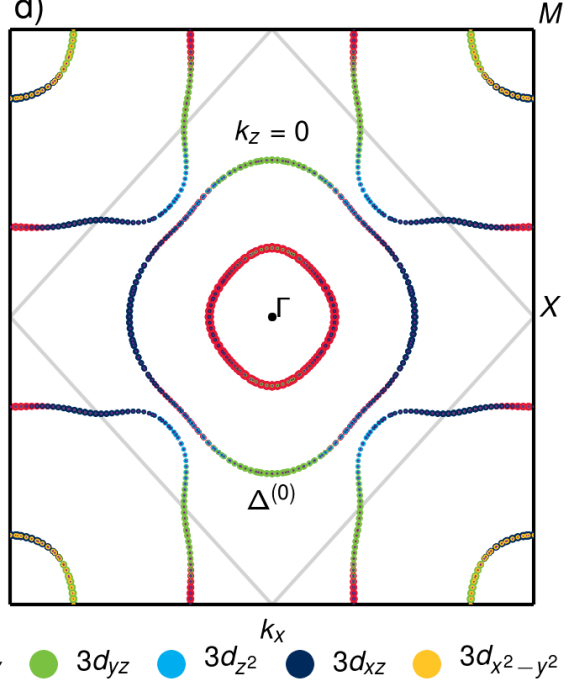

e)

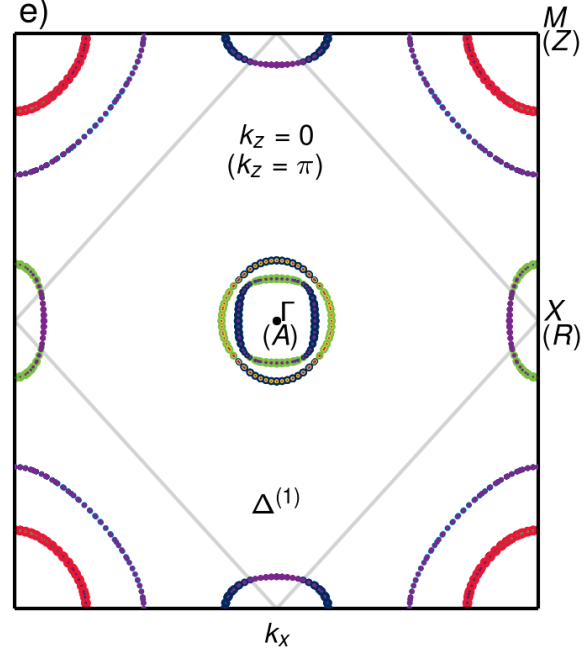

$4 p$

FIG. 7: Unfolding of $\mathrm{CaFe}_{2} \mathrm{As}_{2}$ bandstructure to one iron equivalent Brillouin zone. (a) Bandstructure of $\mathrm{CaFe}_{2} \mathrm{As}_{2}$ in the two-iron Brillouin zone. (b) The unfolded bandstructure obtained from $\Delta^{(\boldsymbol{k}, 0)}$ in the one-iron equivalent Brillouin zone. (c) $k_{z}=0$ cut of $\mathrm{CaFe}_{2} \mathrm{As}_{2}$ Fermi surface cut in the two-iron Brillouin zone. (d) and (e) Projection of the Fermi surface obtained from $\Delta^{(\boldsymbol{k}, 0)}$ and $\Delta^{(\boldsymbol{k}, 1)}$, respectively. Labels enclosed in brackets on panel (e) pertain to $k_{z}=\pi$ cut of the Fermi surface.

Furthermore, our discussion of effects of highsymmetry elements of the Brillouin zone on the interpretation of ARPES data shows that under certain conditions it is possible to observe the spectral function consistent with the one iron picture in $k_{z}=0$ or $k_{z}=\pi$ planes. This is corroborated by recent observations 33. in $\mathrm{CsFe}_{2} \mathrm{As}_{2}$ whose electronic structure is weakly dispersive in the $k_{z}$ direction and the glide mirror unfolding can be accurately extended across the entire Brillouin zone.

The unfolding process also offers a simple answer to the question why the neutron scattering intensities seem to indicate a scenario consistent with the one-iron picture [34, 35. This can be naturally interpreted as a consequence of the fact that neutron scattering intensities are momentum-resolved in the high symmetry plane and transitions between states belonging to different irreducible subspaces are suppressed there.

\section{SUMMARY}

In summary, we have demonstrated with a grouptheoretical treatment of the bandstructure that unfolding can be understood as a projection onto induced irreducible representations of the supergroup of the original translation group. The unfolded Brillouin zone arises as a consequence of the fact that different induced irreducible representations become identical when shifted by an appropriate vector in the Brillouin zone. Due to the projective definition, the unfolding procedure can be generalized for arbitrary quantities in reciprocal space. Also, the unfolding artifacts in the cases where the unfolding is inexact arise because bands have nonzero projections onto multiple irreducible representations.

When point group operations are used, the unfolding is exact only in the high-symmetry k-points of the Brillouin zone. It is nonetheless possible to extend the unfolding to the entire Brillouin zone as long as the bandstructure is 


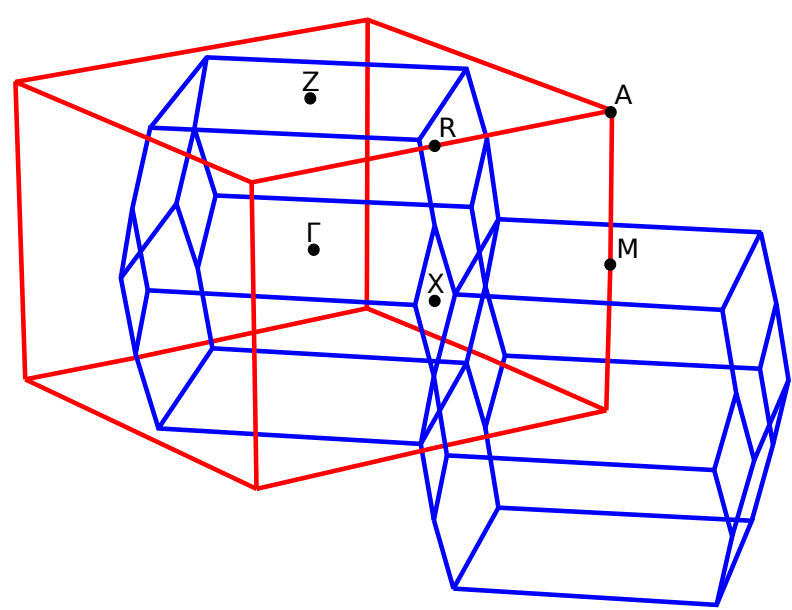

FIG. 8: One and two iron Brillouin zones for the $I 4 / \mathrm{mmm}$ spacegroup. Two adjacent Brillouin zones corresponding to the two iron primitive cell of $I 4 / \mathrm{mmm}$ space group are shown in blue color. The simple tetragonal unit cell corresponding to the one iron unit cell is shown in red.

dominantly dispersive only along the corresponding highsymmetry lines or planes in the Brillouin zone. By making sure this constraint is satisfied, it is possible to formulate tight-binding models of reduced dimensionality without loss of accuracy. In the cases where this is not completely possible, the unfolding framework provides a systematic way to make controlled approximations by projecting the relevant quantities into appropriate irreducible subspaces.

For FeSe under pressure, we have shown how an 8-band tight-binding model can be constructed by unfolding the 16-band tight-binding model with the help of glide-mirror operations. The resulting unfolded model produces the almost exactly unfolded Fermi surface. This results from the fact that the Fermi surface in FeSe is dominated by the iron orbital character. This is in fact the most important requirement that needs to be fulfilled for the one iron model to be an accurate representation of the physics of iron based superconductors. An additional requirement stems from the fact that the one iron picture is formulated as an irreducible representation, and as such for any realistic computation using the one iron model, the off-diagonal elements of the involved observables, connecting the two irreducible representations of the glidemirror group need to be small compared to the diagonal elements.

And finally, we show that careful interpretation of ARPES data in cases where the direct comparison with density functional theory calculations is not immediately obvious, requires consideration of possible effects of high symmetry elements of the Brillouin zone as well as comparison with all irreducible representations arising from the unfolding process.
We would like to thank Doug J. Scalapino, Peter J. Hirschfeld and Lilia Boeri for useful discussions and we gratefully acknowledge financial support by the Deutsche Forschungsgemeinschaft through grant SPP 1458.

* Email tomic@itp.uni-frankfurt.de

[1] P. Hohenberg and W. Kohn, Phys. Rev. 136, B864 (1964).

[2] F. Bloch, Z. Physik 52, 555 (1928).

[3] T. B. Boykin and G. Klimeck, Phys. Rev. B 71, 115215 (2005).

[4] T. B. Boykin, N. Kharche, and G. Klimeck, Phys. Rev. B 76, 035310 (2007).

[5] T. B. Boykin, N. Kharche, G. Klimeck, and M. Korkusinski, J. Phys. Condens. Matter 19, 036203 (2007).

[6] W. Ku, T. Berlijn and C.-C Lee, Phys. Rev. Lett. 104, 216401 (2010).

[7] V. Popescu and A. Zunger, Phys. Rev. B 85, 085201 (2012).

[8] P. V. C. Medeiros, S. Stafström, and J. Björk, Phys. Rev. B 89, 041407(R) (2014).

[9] H. Huang, F. Zheng, P. Zhang, J. Wu, B-L. Gu and W. Duan, New J. Phys. 16, 033034 (2014).

[10] M. X. Chen and M. Weinert, Nano Lett. Article ASAP, doi:10.1021/nl502107v (2014).

[11] E. P. Wigner, Gruppentheorie und ihre Anwendung auf die Quantenmechanik der Atomspektren, Vieweg (1931).

[12] O. K. Andersen and L. Boeri, Ann. Phys. 523, 8 (2011).

[13] C.-H. Lin, T. Berlijn, L. Wang, C.-C. Lee, W.-G. Yin and W. Ku, Phys. Rev. Lett. 107, 257001 (2011).

[14] M. El-Batanouny and F. Wooten, Symmetry and Condensed Matter Physics; A Computational Approach, Cambridge University Press (2008).

[15] S. K. Kim, Group Theoretical Methods and Applications to Molecules and Crystals, Cambridge University Press (1999).

[16] A. Damascelli, Phys. Scr. T109, 61 (2004).

[17] S.-H. Lee, Guangyong Xu, W. Ku, J. S. Wen, C. C. Lee, N. Katayama, Z. J. Xu, S. Ji, Z. W. Lin, G. D. Gu, H.B. Yang, P. D. Johnson, Z.-H. Pan, T. Valla, M. Fujita, T. J. Sato, S. Chang, K. Yamada, and J. M. Tranquada Phys. Rev. B 81, 220502(R) (2010).

[18] G. Borstel1984, Appl. Phys. A 38, 193 (1985).

[19] W. Nolting, J. Braun, G. Borstel, and W. Borgiel, Phys. Scr. 41, 601 (1990).

[20] M. S. Torikachvili, S. L. Budko, N. Ni, and P. C. Canfield, Phys. Rev. Lett. 101, 057006 (2008).

[21] A. Kreyssig, M. A. Green, Y. Lee, G. D. Samolyuk, P. Zajdel, J. W. Lynn, S. L. Bud'ko, M. S. Torikachvili, N. Ni, S. Nandi, J. B. Leão, S. J. Poulton, D. N. Argyriou, B. N. Harmon, R. J. McQueeney, P. C. Canfield, and A. I. Goldman, Phys. Rev. B 78, 184517 (2008).

[22] Y-Z. Zhang, H. C. Kandpal, I. Opahle, H. O. Jeschke and R. Valentí, Phys. Rev. B 80, 094530 (2009).

[23] M. Tomić, R. Valentí and H. O. Jeschke, Phys. Rev. B 85, 094105 (2012).

[24] M. Tomić, H. O. Jeschke, R. M. Fernandes, and R. Valentí, Phys. Rev. B 87, 174503 (2012).

[25] R. S. Dhaka, R. Jiang, S. Ran, S. L. Bud'ko, P. C. Canfield, B. N. Harmon, A. Kaminski, M. Tomić, R. Valentí, 
and Y. Lee, Phys. Rev. B 89, 020511(R) (2014).

[26] K. Gofryk, B. Saparov, T. Durakiewicz, A. Chikina, S. Danzenbächer, D. V. Vyalikh, M. J. Graf, and A. S. Sefat, Phys. Rev. Lett. 112, 186401 (2014).

[27] S. Kasahara, T. Shibauchi, K. Hashimoto, Y. Nakai, H. Ikeda, T. Terashima, and Y. Matsuda, Phys. Rev. B 83, 060505(R) (2011).

[28] K. Koepernik and H. Eschrig, Phys. Rev. B 59, 1743 (1999).

[29] S. Graser, T. A. Maier, P. J. Hirschfeld and D. J. Scalapino, New J. Phys. 11, 025016 (2009)

[30] V. Cvetković and O. Vafek, Phys. Rev. B 88, 134510 (2013).

[31] M. Casula and S. Sorella, Phys. Rev. B 88, 155125 (2013).

[32] C.-H. Lin, C.-P. Chou, W.-G. Yin, W. Ku,
arXiv:1403.3687 (unpublished).

[33] S. Kong, D. Y. Liu, S. T. Cui, S. L. Ju, A. F. Wang, X. G. Luo, L. J. Zou, X. H. Chen, G. B. Zhang, Z. Sun, arXiv:1409.2300 (unpublished).

[34] M. D. Lumsden, A. D. Christianson, E. A. Goremychkin, S. E. Nagler, H. A. Mook, M. B. Stone, D. L. Abernathy, T. Guidi, G. J. MacDougall, C. de la Cruz, A. S. Sefat1, M. A. McGuire, B. C. Sales, and D. Mandrus, Nat. Phys. 6, 182 (2010).

[35] J. T. Park, D. S. Inosov, A. Yaresko, S. Graser, D. L. Sun, Ph. Bourges, Y. Sidis, Yuan Li, J.-H. Kim, D. Haug, A. Ivanov, K. Hradil, A. Schneidewind, P. Link, E. Faulhaber, I. Glavatskyy, C. T. Lin, B. Keimer, and V. Hinkov, Phys. Rev. B 82, 134503 (2010). 